\title{
Plasma chemistry of the chinstrap penguin Pygoscelis antarctica during fasting periods: a case of poor adaptation to food deprivation?
}

\begin{abstract}
The chinstrap penguin (Pygoscelis antarctica) is the smallest penguin species to be used to study the physiology of fasting. We analysed body-mass change and plasma chemistry of five non-breeding chinstraps during an experimental fasting period in the breeding season. We also analysed the same parameters in six fasting birds under natural conditions (during an incubation shift, which lasts about 10 days). Both groups presented similar patterns of change, showing a rapid increase in urea and uric acid plasma concentrations. Urea surpassed $3 \mathrm{mmol} / \mathrm{l}$ after 5 fasting days, while uric acid reached $1 \mathrm{mmol} / \mathrm{l}$ after 9 days. Plasma glucose levels decreased after 11 days, whereas cholesterol also showed a clear reduction during fasting. These results as a whole suggest that chinstrap penguins reached phase III after a short period in comparison with other Pygoscelis species. Body size and ecological factors could explain these inter-specific differences.
\end{abstract}

\section{Introduction}

The extreme weather conditions in the polar regions are unique on Earth. Animals have adapted to these extremes by means of a variety of strategies such as long fasting periods during reproduction. Fasting physiology has been extensively studied in two penguin species, the emperor penguin (Aptenodytes forsteri) and the king

C. Alonso-Alvarez $(\boldsymbol{\&}) \llbracket \mathrm{M}$. Ferrer $₫$ J.A. Amat

Estacion Biologica de Donana, CSIC,

Avd. Marıa Luisa, Pabellon del Peru, 41013 Seville, Spain

J. Vinuela

Museo Nacional de Ciencias Naturales,

CSIC, J. Gutierrez Abascal 2, 28006 Madrid, Spain

Present address C. Alonso-Alvarez

Laboratoire d'Parasitologie Evolutive,

Universite Pierre et Marie Curie, 7 quai St. Bernard,

Case 237, 75005 Paris, France,

e-mail: calonso@snv.jussieu.fr penguin (A. patagonicus; e.g. Groscolas 1986; Cherel et al. 1988a; Robin et al. 1988), and has also been assessed in the gentoo penguin (Pygoscelis papua; Cherel et al. 1993). The length of fasting periods during the breeding season is greatly variable among penguin species. Emperors fast for 1-4 months (Williams 1995), kings for up to 54 days (Stonehouse 1960) and macaroni penguins (Eudyptes chrylosophus) may fast for around 40 days (Williams 1995). The Adelie penguin (P. adeliae) fasts for 10-40 days (Ulbricht and Zippel 1994; Bucher and Vleck 1999), whereas gentoos fast for short periods (2-3 days; Cherel et al. 1993). This variability suggests species-specific differences in fasting physiology.

Three fasting phases based on changes in body mass, protein and lipid reserves have been proposed in penguins (e.g. Cherel et al. 1988b; Robin et al. 1988) and other birds (e.g. Handrich et al. 1993; Sartori et al. 1995). In the first phase (named phase I), body mass shows a significant reduction in a short period of time. Phase II presents a slow and stable daily weight descent over a period that can vary depending on the capacity of each species to support fasting periods. Finally, phase III consists of a quick increase in body-mass loss that reaches a critical level close to death (a recent review in Cherel and Groscolas 1999).

In species adapted to fasting, protein is the fuel whose exhaustion determines survival, given its importance in body structure and muscle function (Cherel et al. 1988a; Castellini and Rea 1992; Groscolas and Robin 2001). The plasma nitrogen wastes derived from protein catabolism (urea and uric acid) decrease during phase I, maintain a low and stable concentration throughout phase II, and rise suddenly to reach their highest concentrations during phase III (use of muscle proteins as an energy source). This last increment precedes the bird's death or, as in the case of penguins, can lead to the abandonment of breeding duties in order to re-feed ("refeeding signal"; Cherel et al. 1988b; Castellini and Rea 1992; Groscolas and Robin 2001).

However, in birds, the largest reservoir of body fuel is in the form of fat, stored as triglycerides (Griminger 

1986). Plasma triglycerides regularly decrease during fasting (Jenni-Eiermann and Jenni 1994), though they were not analysed in this three-phase model. Cholesterol is another lipid that has been recently related to fasting periods because it shows a strong correlation to bodymass change, decreasing during fasting probably as a consequence of a reduction in the anabolic activity (Alonso-Alvarez and Ferrer 2001). Finally, plasma glucose maintains its levels, only decreasing in phase III (Cherel et al. 1988b; Boismenu et al. 1992). This carbohydrate is a critical fuel source for the central nervous system and, therefore, its circulating level is tightly controlled (Castellini and Rea 1992).

In the present study, the physiological adaptation of chinstrap penguins ( $\mathrm{P}$. antarctica) to fasting was analysed by means of plasma biochemistry. This is the smallest penguin species to have been used to study fasting physiology (range 3-5 kg; Williams 1995). As they wait for their mates to take over incubation duties (during their "incubation shift"), they may have to endure around 6-10 days of fasting (Williams 1995). This supposes a fasting length intermediate between the other two Pygoscelis species (see above), although gentoos showed a strong capacity to endure experimentally prolonged fasting (13 days, Mougin 1972; 12 days, Cherel et al. 1993). We analysed variations in plasma chemistry composition and body mass in captive and free-living incubating chinstraps and showed changes in enzymes and other plasma parameters. Although these changes may not directly reflect body composition (e.g. Brown 1996; Stevens 1996), they have been extensively used to describe the state of fat or protein reserves and to analyse the avian fasting process (e.g. Castellini and Rea 1992; Alonso-Alvarez and Ferrer 2001; Groscolas and Robin 2001).

\section{Materials and methods}

This study was carried out on Deception Island, South Shetland Islands $\left(62^{\circ} 55 \mathrm{cS}, 60^{\circ} 46 \mathrm{cW}\right)$, at the beginning of December during the 1990-1991 Antarctic summer, when penguins were not moulting, to thus eliminate changes in blood metabolites related to feather production (see Cherel et al. 1988c; Groscolas and Cherel 1992; Cherel et al. 1994). Three male and two female non-breeding adult chinstrap penguins were captured outside the rookeries (sexed according to Amat et al. 1993). Non-breeding adults were easily identified by the absence of incubation patches and by their peripheral position in the colony.

To consider the possibility of a previous bad body condition of these individuals, we compared them with another 138 incubating birds (65 males and 73 females). Their body masses (males: 4,080 and 4,220 g; females: 3,340, 3,500 and 3,880 g) were higher than the mean values for the breeders (males: 3,909 $\mathrm{g}$, females: 3,300 g). Moreover, the body masses corrected by differences in body size were compared. A linear regression between log-transformed mass and bill length was performed, including both the mass of the 138 birds and the 1st-day mass of our experimental birds $\left(\mathrm{R}^{2}=0.38\right.$, $\mathrm{P}=0.001$, and $\mathrm{R}^{2}=0.35, \mathrm{P}=0.004$, males and females respectively; see e.g. Brown 1996). The residuals of the non-breeders were positive (range: $+0.02-+0.17$ ).

The captured penguins were kept outdoors in a fenced area, snow being given daily ad libitum as a water source. In order to avoid stress, this area was situated in an isolated place and only visited once a day. From capture onwards, birds were not fed for 11 days (experimental fasting). Originally, a 14-day fasting period had been intended, based on data from 8 birds that had deserted after 14 days fasting during incubation (unpublished observations). However, in order to avoid death, the penguins were re-fed after 11 days, since they had reached very high urea and uric acid plasma values, as well as lower glucose levels than those described for the species (Aguilera et al. 1993; Ferrer et al. 1994; blood samples were immediately analysed; see below). After the experiment ended, birds were treated with intravenous glucose and liberated, and regularly sighted in the colony over the period of a month. We used small sample sizes in order to avoid imposing unnecessary suffering on more individuals (Still 1982). In addition, six free-living incubating birds (three males and three females) were sampled in order to validate the study. The number of days these birds had already spent fasting before sampling was known.

Captive penguins were measured and weighed the day after capture (day 1) and, thereafter, weighed every other day. We recorded body mass using a spring balance (accuracy $10 \mathrm{~g}$ ). The variable Cumulative Body-Mass Loss was defined as the proportion of body mass lost relative to the weight at the beginning of the experiment. The Relative Body-Mass Loss represented the change in body weight with respect to the previous sample and allowed us to observe small changes during 2-day periods.

Blood samples of captive individuals were also collected every other day from day 1 onwards (always before weighing). All blood samples were drawn between 1100 and 1400 hours (solar time) to eliminate possible diurnal fluctuations caused by circadian rhythms (Ferrer et al. 1994). We extracted up to $2 \mathrm{ml}$ of blood from the brachial vein. Blood was collected in lithium heparin tubes. Centrifugation and plasma separation (10 $\mathrm{min}$ at 3,000 rpm) were done less than $6 \mathrm{~h}$ after the sample was drawn. Blood analyses were carried out in a portable autoanalyser (Reflotron II, with the reagents recommended by Boehringer-Mannheim). Uric acid, urea, triglycerides, glucose and cholesterol were determined. Triglycerides analyses included amounts of free glycerol (following McGowan et al. 1983). All analyses were carried out less than $8 \mathrm{~h}$ after the blood extraction.

Non-parametric statistics were used conservatively due to the small sample size. Since experimental data were dependent on each bird, Wilcoxon matched-pairs signed-ranks test was applied in order to analyse within-subject differences between two sampling days, while Friedman's test was used to determine within-subject change throughout the experiment.

\section{Results}

The mean body mass of captive penguins at day 1 was $3,804 \mathrm{~g}$. At the end of the experiment, $31.8 \%$ of their initial weight had been lost (mean value; range $24.9-43.4 \%$ ). Cumulative body-mass loss regularly increased during fasting (Friedman's test: $\mathrm{v}^{2}=20.0, \mathrm{df}=\mathbf{4}$, $\mathrm{P}<0.001$ ), whereas relative body-mass loss showed a stable pattern $\left(\mathrm{v}^{2}=0.96, \mathrm{df}=4, \mathrm{P}=0.960\right.$; mean $7.5 \%$, range 1.1-12.2\%; Fig. 1).

Plasma uric acid and urea values clearly increased throughout fasting (Table 1; Fig. 2). However, urea concentration showed a decrease from the 1st to the 3rd day in captive birds (Wilcoxon matched-pairs signedranks test: $\mathrm{Z}=2.02, \mathrm{P}<0.05)$ and uric acid showed a tendency to statistical significance in the same direction $(\mathrm{Z}=1.75$, $\mathrm{P}=0.08$; Fig. 2). Triglycerides levels showed an unstable pattern during the experimental fasting. There was no significant difference between concentrations on the first and last days, although there was 

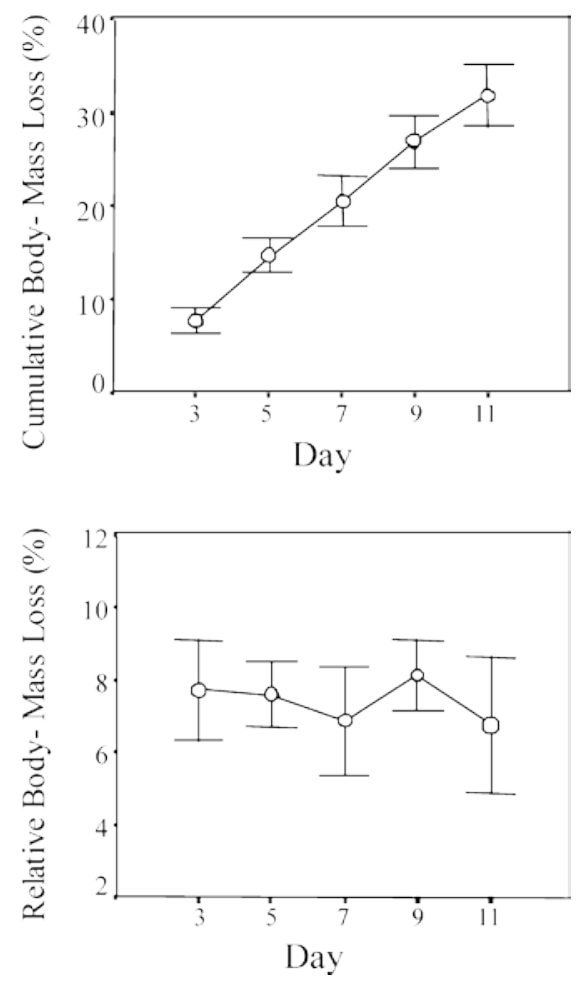

Fig. 1 Proportions of body-mass change (means \pm SE) calculated with respect to initial values on day 1 (above) and with respect to the values on each previous sampling day (below; 2-day intervals) during experimental fasting (11 days) in 5 chinstrap penguins

variation throughout the whole experiment (Table 1). Thus, this lipid showed an increase during the first days (day 1 vs day 3: $\mathrm{P}<0.05$ ), decreasing to initial levels by day 9 (day 7 vs day 9: $P<0.05$ ), and again increasing on the last day of sampling (day 9 vs day 11: $\mathrm{P}<0.05$ ). Glucose was more or less stable during the first 9 days, descending on the last day (Fig. 2). Cholesterol plasma concentration decreased during the experiment. Although there was no significant change between initial and final cholesterol values (Table $1 ; \mathrm{P}=0.08$ ), there were differences between the 3rd day and last day values, and between the 1st and 3rd days $(\mathrm{P}<0.05$; Fig. 2). The fasting penguins incubating naturally showed nearly identical patterns and values as compared to the experimental birds (see Fig. 2).

\section{Discussion}

Chinstrap penguins seem to show poorer adaptation to fasting than other analysed penguins (i.e. Cherel et al. 1988b; Robin et al. 1988; Cherel et al. 1993). Plasma levels of nitrogen wastes and glucose suggest that chinstraps reached phase III of fasting in only a few days. Although they showed a lower cumulative body-mass loss proportion than the larger emperor penguins (52\%; Robin et al. 1988) or king penguins (47\%; Cherel et al. 1988b) in phase III, they showed higher values at day 11 than another Pygoscelis penguin (gentoo) after 12 days
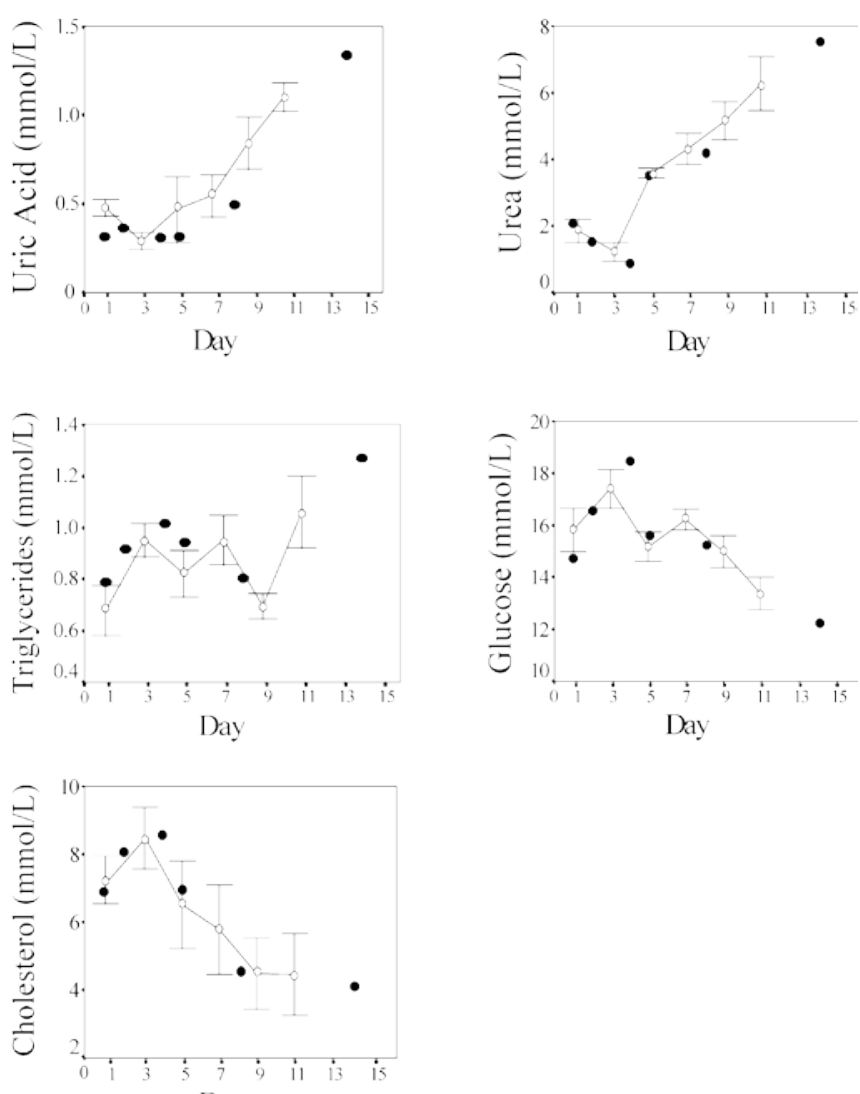

Day

Fig. 2 Change in plasma biochemical parameters in 5 chinstrap penguins during experimental fasting (11 days; unfilled circles and bars: means \pm SE) and also in 6 incubating chinstraps during natural fasting (filled circles: days 1, 2, 4, 5, 8 and 14)

of experimental fasting (mean 23\%; Cherel at al. 1993). Moreover, the closely related Adelie penguin (see Lishman 1985; Trivelpiece et al. 1987; Williams 1995) loses a similar body-mass proportion to chinstraps, but only after longer periods $(25 \%$ and $39 \%$ after 30 and 45 days, in males and females respectively; Vleck and Bucher 1999). The relative body-mass loss was regular and did not increase towards the end of the experiment, as predicted by the three-phase fasting pattern. However, the sampling interval (2 days) may not have adequately detected the changes occurring.

Furthermore, plasma levels of urea showed an initial decrease (predicted in phase I), whereas both urea and uric acid significantly increased after 5 and 9 days, respectively (phase III). Moreover, similar concentrations were observed in the free-living individuals during their incubation shifts. These high values are only reached in other avian species after a long fasting period (king penguin: 1 month, Cherel et al. 1988b; emperor penguin: 80 days, Robin et al. 1988; greater snow geese Chen caerulescens atlantica: 1 month, Boismenu et al. 1992). Cherel et al. (1993) calculated that gentoo penguins would reach phase III after 17 days. They reported only $0.25 \mathrm{mmol} / \mathrm{l}$ after 11-14 days for experimental fasting gentoos. Hence, the present results 
Table 1 Plasma concentration of 5 parameters in 5 captive chinstrap penguins on the 1 st, 5 th and last days (day 11) of experimental fasting

\begin{tabular}{|c|c|c|c|c|c|c|c|c|}
\hline \multirow[t]{2}{*}{ Parameter } & \multicolumn{2}{|c|}{ First Day } & \multicolumn{2}{|c|}{ Fifth Day } & \multicolumn{2}{|c|}{ Last Day } & \multirow{2}{*}{$\begin{array}{l}\text { First versus } \\
\text { last day } \\
\mathrm{Z}\end{array}$} & \multirow{2}{*}{$\begin{array}{l}\text { Whole } \\
\text { fasting } \\
v^{2}\end{array}$} \\
\hline & Mean & Range & Mean & Range & Mean & Range & & \\
\hline Uric acid $(\mathrm{mmol} / \mathrm{l})$ & 0.47 & $(0.36-0.59)$ & 0.46 & $(0.15-1.18)$ & 1.10 & $(0.85-1.27)$ & $2.02^{*}$ & $18.71^{* *}$ \\
\hline Urea $(\mathrm{mmol} / \mathrm{l})$ & 1.84 & $(0.87-2.70)$ & 3.59 & $(3.05-3.88)$ & 6.32 & $(4.80-9.27)$ & $2.02^{*}$ & $24.54^{* * *}$ \\
\hline Triglycerides $(\mathrm{mmol} / \mathrm{l})$ & 0.71 & $(0.52-1.05)$ & 0.85 & $(0.6-1.1)$ & 1.11 & $(0.82-1.54)$ & 1.48 & $15.4^{* *}$ \\
\hline Glucose $(\mathrm{mmol} / \mathrm{l})$ & 15.13 & $(12.94-16.17)$ & 15.01 & $(12.9-16.76)$ & 13.34 & $(11.67-14.44)$ & $2.02^{*}$ & $19.51^{* *}$ \\
\hline Cholesterol $(\mathrm{mmol} / \mathrm{l})$ & 7.18 & $(5.39-9.97)$ & 7.21 & $(4.07-11.6)$ & 4.37 & $(2.28-7.74)$ & 1.75 & $15.17^{* *}$ \\
\hline
\end{tabular}

Wilcoxon matched-pairs signed-ranks test was calculated to analyse changes in individual values between the first and last day. Friedman's test was used to study changes throughout 11 days of fasting (sampled each 2 days; $\mathrm{df}=5,{ }^{*} \mathrm{P}<0.05 ;{ }^{* *} \mathrm{P}<0.01 ;{ }^{* *} \mathrm{P}<0.001$ )

suggest that chinstraps reached the last fasting phase quickly.

Plasma triglycerides in birds are positively correlated to the amount of body fat (Bacon et al. 1989; Dabbert et al. 1997) and regularly decrease during fasting (Didier et al. 1983; Jenni-Eiermann and Jenni 1994). Surprisingly, in chinstrap penguins this lipid first increased, declining to initial values after 9 days. In our study, triglycerides concentrations also included amounts of free glycerol. Triglycerides are the precursors of glycerol, free fatty acids and ketone bodies (Griminger 1986). The plasma level of these substances increases during fasting in phase I due to triglyceride breakdown, maintaining high values in phase II and abruptly decreasing in phase III (exhaustion of fat stores; e.g. Cherel et al. 1988a). Therefore, the observed changes in plasma triglycerides might reflect fat reserve mobilisation. The same quadratic pattern of changes (increase followed by a decrease) was found in yellow-legged gulls (Larus cachinnans) during an experimental fasting period (using the same analysis method; Alonso-Alvarez and Ferrer 2001). Finally, triglycerides levels increased on the last fasting day. This might reflect some damage to the organism, such as nephrosis or biliary obstructions (e.g. Fredrickson et al. 1967), although more information would have been needed to confirm this.

The glucose concentration decreased on the last day of fasting (lower levels than those reported by Aguilera et al. 1993 and Ferrer et al. 1994 in chinstraps). Although several authors have detected an increment of plasma glucose in birds during phase III (Hazelwood and Lorenz 1959; Groscolas 1986; Garcia-Rodriguez et al. 1987), the majority have described a stable pattern throughout fasting, only decreasing close to death (e.g. Langslow 1978; Cherel et al. 1988b; Boismenu et al. 1992). The maintenance of plasma glucose during fasting depends primarily on gluconeogenesis from tryglycerides (e.g. Stevens 1996), and afterwards from the amino acids that are obtained from protein breakdown (Davison and Langslow 1975; Watford 1985). The early increase in blood nitrogen wastes suggests an early use of these amino acids as glycogenic substrate. This could have contributed to a reduced capacity to maintain plasma glucose levels at the end of the fasting period.
Cholesterol may provide supplementary information about fasting physiology. In yellow-legged gulls, cholesterol level was the strongest correlated parameter with cumulative body-mass loss during fasting (Alonso-Alvarez and Ferrer 2001; Alonso-Alvarez et al. 2002). This lipid is involved in the formation of cellular membranes, steroids and bile acids (Griminger 1986). The plasma cholesterol level decreased from the 3rd day of fasting in chinstraps. Hence, this result might reflect the reduction of some anabolic processes. However, plasma cholesterol increased during the first few days. The stress produced by food deprivation might explain this variation. In fact, plasma cholesterol may increase in birds during periods of stress (Freeman and Manning 1976; Puvadolpirod and Thaxton 2000) and during short fasting periods (Jeffrey et al. 1985; Loo et al. 1990).

In summary, our results showed that chinstraps reach phase III in a short time period with respect to other studied penguin species. The chinstrap is the smallest penguin species yet analysed in any study on long-term fasting physiology. The other previously analysed Pygoscelis penguin, the gentoo, seems to show a poorer adaptation to food-deprivation than Aptenodytes penguins, but nevertheless still better than that of chinstraps (Cherel et al. 1993). The gentoo is the largest and heaviest penguin species other than the king and emperor penguins (Williams 1995) and is about $1-2 \mathrm{~kg}$ heavier than chinstraps (Cherel et al. 1993; Williams 1995). The lower energy consumption of larger species could facilitate better reserve allocation (i.e. Croxall 1982), although gentoos do not undergo long fasting periods under natural conditions. Heavy body mass and the large size of gentoos could have evolved as an adaptation to diving (see Trivelpiece et al. 1987; Wilson 1995) or in an unknown ecological context and, at the present, these features provide them with a good fasting capacity.

Nevertheless, the other Pygoscelis species (Adelie penguin) has a similar body size and longer fasting periods than chinstraps. Lishman (1985) considered the difference in the length of chinstraps' and Adelies' fasting periods to be the result of competition for food. Adelies start breeding about 1 month before chinstraps, thereby enduring lower temperatures but avoiding feeding interference. In contrast, Trivelpiece et al. (1987) 
suggested that the length of incubation shifts in Pygoscelis penguins could be the result of an adaptation to the environmental conditions in the centre of their respective geographic distributions. In conclusion, the origin of fasting adaptations in Pygoscelis penguins remains unclear, and so the fasting physiology of the Adelie penguin should be analysed in future studies in an attempt to complete the scenario.

Acknowledgements Logistic support was offered by the base Gabriel de Castilla (Spanish Army), and transport by the boat Las Palmas (Spanish Navy). We thank Alberto Velando for his peer review of an early draft of this manuscript, and Mike Lockwood for the English review. The capture and manipulation of birds, as well as the experiment, were conducted with Spanish legal permission, avoiding unnecessary damage to the birds.

\section{References}

Aguilera E, Moreno J, Ferrer M (1993) Blood chemistry values in three Pygoscelis penguins. Comp Biochem Physiol 105A: 471-473

Alonso-Alvarez C, Ferrer M (2001) A biochemical study about fasting, subfeeding and recovery processes in yellow-legged gulls. Physiol Biochem Zool 74:703-713

Alonso-Alvarez C, Ferrer M, Velando A (2002) The plasmatic index of body condition in yellow-legged Gulls (Larus cachinnans): a food-controlled experiment. Ibis 144:147-149

Amat JA, Vinuela J, Ferrer M (1993) Sexing chinstrap penguins (Pygoscelis antarctica) by morphological measurements. Colon Waterbird 16:213-215

Bacon WL, Nestor KE, Naber EC (1989) Prediction of carcass composition of turkeys by blood lipids. Poultry Sci 68:12821288

Boismenu C, Gauthier G, Larrochelle J (1992) Physiology of prolonged fasting in greater snow geese (Chen caerulescens atlantica). Auk 109:511-521

Brown ME (1996) Assessing body condition in birds. In: Nolan V, Ketterson ED (eds) Current ornithology, vol 13. Plenum Press, New York, pp 67-135

Bucher TL, Vleck CM (1999) Patterns of nest attendance and relief in Adelie penguins, Pygoscelis adeliae. Antarct J U S 32:105107

Castellini MA, Rea LD (1992) The biochemistry of natural fasting at its limits. Experientia 48:575-582

Cherel Y, Groscolas R (1999) Relationships between nutrient storage and nutrient utilisation in long-term fasting birds and mammals. In: Adams NJ, Slotow RH (eds) Proceedings 22 International Ornithology Congress, Durban. BirdLife South Africa, Johannesburg

Cherel Y, Robin JP, Le Maho Y (1988a) Physiology and biochemistry of long-term fasting in birds. Can J Zool 66:159-166

Cherel Y, Leloup J, Le Maho Y (1988b) Fasting in king penguin. I. Hormonal and metabolic changes during breeding. Am J Physiol 254:R170-R177

Cherel Y, Leloup J, Le Maho Y (1988c) Fasting in king penguin. II. Hormonal and metabolic changes during molt. Am J Physiol 254:R178-R184

Cherel Y, Freby F, Gilles J, Robin J-P (1993) Comparative fuel metabolism in gentoo and king penguins: adaptation to brief versus prolonged. Polar Biol 13:263-269

Cherel Y, Charrassin J-B, Challet E (1994) Energy requirements for molt in the king penguin Aptenodytes patagonicus. Am J Physiol 266:R1182-R1188

Croxall JP (1982) Energy costs of incubation and moult in petrels and penguins. J Anim Ecol 51:177-194

Dabbert CB, Martin TE, Powell KC (1997) Use of body measurements and serum metabolites to estimate the nutritional status of mallards wintering in the Mississipi Alluvial Valley, USA. J Wildlife Dis 33:57-63

Davison TF, Langslow DR (1975) Changes in plasma glucose and liver glycogen following the administration of gluconeogenic precursors to the starving fowl. Comp Biochem Physiol 52A:645-649

Didier R, Remesy C, Demigne C (1983) Changes in glucose and lipid metabolism in starved or starved-refed Japanese quail (Coturnix coturnix japonica) in relation to fine structure of liver cells. Comp Biochem Physiol A74:839-848

Ferrer M, Amat JA, Vinuela J (1994) Relative variations of blood chemistry values in the chinstrap penguin (Pygoscelis antarctica) during the Antartic summer. Comp Biochem Physiol 107A:81-84

Fredrickson DS, Levy IR, Lees RS (1967) Fat transport and lipoproteins - an integrated approach to mechanisms and disorders. N Engl J Med 276:273-281

Freeman BM, Manning AC (1976) Mediation of glucagon in the response of the domestic fowl to stress. Comp Biochem Physiol 53A:169-171

Garcia-Rodriguez T, Ferrer M, Carrillo JC, Castroviejo J (1987) Metabolic responses of Buteo buteo to long-term fasting and refeeding. Comp Biochem Physiol 87A:381-386

Griminger P (1986) Lipid metabolism. In: Sturkie PD (ed) Avian physiology. Springer, Berlin Heidelberg New York, pp 345-358

Groscolas R (1986) Changes in body mass, body temperature and plasma fuel levels during the natural breeding fast in male and female emperor penguins Aptenodytes forsteri. J Comp Physiol B 156:521-527

Groscolas R, Cherel Y (1992) How to molt while fasting in the cold: the metabolic and hormonal adaptations of emperor and king penguins. Ornis Scand 23:328-334

Groscolas R, Robin J-P (2001) Long-term fasting and re-feeding in penguins. Comp Biochem Physiol A 128:645-655

Handrich Y, Nicolas L, Le Maho Y (1993) Winter starvation in captive barn-owls: physiological states and reversible limits. Auk 110:458-469

Hazelwood RL, Lorenz FW (1959) Effects of fasting and insulin on carbohydrate metabolism of the domestic fowl. Am J Physiol 197:47-51

Jeffrey DA, Peakall DB, Miller DS, Herzberg GR (1985) Blood chemistry changes in food-deprived herring gulls. Comp Biochem Physiol 81A:911-913

Jenni-Eiermann S, Jenni L (1994) Plasma metabolite levels predict individual body mass changes in a small long-distance migrant, the garden warbler. Auk 111:888-899

Langslow DR (1978) Gluconoegenesis in birds. Biochem Soc Trans 6:1148-1152

Lishman GS (1985) The comparative breeding biology of Adelie and chinstrap penguins Pygoscelis adeliae and P. antarctica at Signy Island, Orkney Islands. Ibis 127:84-99

Loo G, Berlin E, Allen PC (1990) Effect of feed deprivation on hepatic membrane and lipoprotein fluidity and binding of lipoproteins to hepatic membranes in the chick (Gallus domesticus). Comp Biochem Physiol 96B:361-366

McGowan MW, Artiss JD, Strandbergh DR, Zak B (1983) A peroxidase-coupled method for the colorimetric determination of serum triglycerides. Clin Chem 29:538-542

Mougin JL (1972) Enregistrements continus de temperatures internes chez quelques Sphenicidae. I. Le Manchot papou Pygoscelis papua de l'lle de la Possession (Archipel Crozet). L'Oiseau RFO Spec Issue 42:84-110

Puvadolpirod S, Thaxton JP (2000) Model of physiological stress in chickens. 1. Response parameters. Poultry Sci 79:363-369

Robin J-P, Frain M, Sardet C, Groscolas R, Le Maho Y (1988) Protein and lipid utilization during long-term fasting in emperor penguins. Am J Physiol 254:R61-R68

Sartori DRS, Migliorini RH, Veiga JAS, Moura JL, Kettelhut IC, Linder C (1995) Metabolic adaptations induced by long term fasting in quails. Comp Biochem Physiol 111A:487-493

Stevens L (1996) Avian biochemistry and molecular biology. Cambridge University Press, Cambridge 
Still AW (1982) On the number of subjects used in animal behaviour experiments. Anim Behav 30:873-880

Stonehouse B (1960) The king penguin Aptenodytes patagonica of South Georgia. I. Breeding behaviour and development. Sci Rep Falkland Isl Depend Surv 23:1-81

Trivelpiece WZ, Trivelpiece SG, Volkman NJ (1987) Ecological segregation of Adelie, gentoo, and chinstrap penguins at King George Island, Antarctica. Ecology 68:351-361

Ulbricht J, Zippel D (1994) Delayed laying and prolonged fasting in Adelie penguins Pygoscelis adeliae. Polar Biol 14:215-217
Vleck CM, Bucher TL (1999) Reproductive biology of free-living Adelie penguins at Torgersen island, Antarctica. Antarct J U S 32:105-107

Watford M (1985) Gluconeogenesis in the chicken: regulation of phosphoenolpyruvate carboxykinase gene expression. Fed Proc Fed Am Soc Exp Biol 44:2469-2474

Williams TD (1995) The penguins. Oxford University Press, Oxford

Wilson RP (1995) Foraging ecology. In: Williams TD (ed) The penguins. Oxford University Press, Oxford, pp 81-106 